\title{
Hypoxic exposure activates the B cell-specific Moloney murine leukaemia virus integration site 1/PI3K/Akt axis and promotes EMT in leukaemia stem cells
}

\author{
MINGYAN JIANG ${ }^{1}$, GUOQIAN HE ${ }^{1}$, JIANHUA LI ${ }^{2}$, JINRONG $\mathrm{LI}^{2}, \mathrm{XIA} \mathrm{GUO}^{2}$ and $\mathrm{JU} \mathrm{GAO}^{1}$ \\ ${ }^{1}$ Department of Pediatric Hematology and Oncology, West China Second University Hospital of \\ Sichuan University; ${ }^{2}$ Key Laboratory of Birth Defects and Related Diseases of Women and Children, \\ Sichuan University, Ministry of Education, Chengdu, Sichuan 610041, P.R. China
}

Received March 5, 2020; Accepted October 26, 2020

DOI: $10.3892 / 01.2020 .12359$

\begin{abstract}
Acute myeloid leukemia (AML) is a malignant tumor of the immature myeloid hematopoietic cells in the bone marrow. Disease recurrence driven by leukaemia stem cells (LSCs), a sub-population of leukaemia cells presenting self-renewal capacity and differentiation potential, is a major problem in the treatment of AML. Although a hypoxic microenvironment is considered to promote AML malignant behaviours and is considered a potential therapeutic target, the effect of hypoxic stimulation of LSCs is still largely unknown. Therefore, the present study analysed the effects of hypoxia on the malignant behaviours of LSCs. Hypoxia exposure upregulated hypoxia-inducible factor (HIF)-1 $\alpha$, which upregulated the transcription of B cell-specific Moloney murine leukaemia virus integration site 1 (BMI-1). Hypoxia exposure also activated the PI3K/Akt pathway and promoted the epithelial mesenchymal transition (EMT) in LSCs via hypoxia-mediated activation of HIF-1 $\alpha$. BMI-1 served an important role in the hypoxia-induced activation of the PI3K/Akt pathway and the promotion of EMT. Hypoxia exposure promoted chemoresistance against cytarabine arabinoside by inducing HIF- $1 \alpha$, thus activating the transcriptional activity of HIF-1 $\alpha$. Knockdown of BMI-1 disrupted hypoxia-induced chemoresistance in LSCs, indicating that HIF-1 $\alpha$-induced BMI-1 has a role in hypoxia-promoted malignant behaviours. Furthermore, it was demonstrated that induced BMI-1 inhibits the self-renewal capacity in LSCs under hypoxic conditions. The present study provides in vitro evidence demonstrating that hypoxia exposure regulates LSCs by activating HIF-1 $\alpha /$ BMI-1 signalling, in
\end{abstract}

Correspondence to: $\mathrm{Dr} \mathrm{Ju}$ Gao, Department of Pediatric Hematology and Oncology, West China Second University Hospital of Sichuan University, No.20 Section 3, South Renmin Road, Chengdu, Sichuan 610041, P.R. China

E-mail: gaoju651220@126.com

Key words: leukaemia stem cells, hypoxia, hypoxia-inducible factor $1 \alpha$, B cell-specific Moloney murine leukaemia virus integration site 1 , epithelial mesenchymal transition turn modulating PI3K/Akt signalling and EMT. These results highlight potentially novel therapeutic targets of LSCs to improve the treatment of AML.

\section{Introduction}

Leukaemia stem cells (LSCs) present similar morphology to normal haematopoietic stem cells and are characterized by their self-renewal capacity and differentiation potential (1). The sub-population of LSCs existing in leukaemia is considered to contribute to leukaemia chemoresistance, relapse and prognosis $(2,3)$. Completely eliminating LSCs and reducing doses of chemotherapeutic agents is one of most promising strategies for improving the prognosis in patients with leukaemia (4). LSCs are also characterized by the surface markers $\mathrm{CD}^{3} 4^{+}$and $\mathrm{CD} 38^{-}$, which are widely expressed in the human leukaemia cell line KG-1 $\alpha(5,6)$. Notably, KG-1 $\alpha$ cells have a similar phenotype of self-renewal capacity and chemoresistance to LSCs, including sensitivity to the widely used chemoagent cytarabine arabinoside (Ara-C) $(5,6)$ and are thus considered an ideal cell model for LSC investigation.

Hypoxia is a critical regulator of tumourigenesis mainly by stimulating angiogenesis through hypoxia-inducible factor (HIF)-1 $\alpha$-mediated upregulation of VEGF. Hypoxia regulates the microenvironment and exerts pro-survival effects on acute myeloid leukaemia (AML) cells by activating the PI3K/Akt pathway via directly upregulating HIF-1 $\alpha$ (7). Moreover, hypoxia also regulates malignant behaviors of LSCs via activating HIF-1 $\alpha$ activity. Zhang et al (8) previously reported that HIF-1 $\alpha$, a master transcriptional regulator responsible for hypoxia exposure, is essential for survival maintenance of LSCs by activating TGF- $\beta$. It has also been demonstrated that HIF-1 $\alpha$ has a protective role for LSCs against oxidative stress under hypoxic conditions, chemotreatment and oncogene transformation (8). Although accumulating evidence suggests that HIF-1 $\alpha$ serves a critical regulatory role in LSCs, the molecular mechanisms by which hypoxia regulates LSCs are still largely unknown $(9,10)$.

Several studies have revealed that B-cell-specific Moloney murine leukaemia virus integration site (BMI)-1 plays an important role in maintaining the self-renewal capacity of 
LSCs, as well as in normal haematopoietic stem cells (11-14). BMI-1 functions as a transcriptional inhibitor and represses the transcription of a range of target genes, including cyclin-dependent kinase inhibitor $2 \mathrm{~A}$ and HOX cluster genes $(15,16)$. It is also reported that BMI-1 is overexpressed in AML and is associated with aggressiveness and poor outcome for patients with AML $(17,18)$. This is possibly due to the hypoxic microenvironment of the bone marrow, where LSCs mainly exist (19). Inhibition of BMI-1 using a preclinical medicine, PTC-209, markedly suppresses colorectal cancer cell growth and eradicates cancer-initiating cells (20). In LSCs, inhibition of BMI-1 induces apoptosis and promotes the phosphorylation of the Akt pathway in $\mathrm{CD} 34^{+} \mathrm{CD} 38^{-}$cells from patients with AML (21). Accordingly, hypoxic stress may regulate LSCs by regulating BMI-1 expression.

The present study investigated the effects of hypoxia on the malignant behaviours of LSCs derived from KG-1 $\alpha$ cells and the mechanisms of action in LSCs. The results provide evidence demonstrating that hypoxia-stimulated HIF-1 $\alpha$ transcriptionally activated BMI-1 and thus activated the PI3K/Akt and EMT pathways.

\section{Materials and methods}

Cell culture and treatment. The human leukaemia cell line KG-1 $\alpha$ bought from the American Type Culture Collection was cultured in RPMI-1640 supplemented with $10 \%$ fetal bovine serum (FBS, Gibco; Thermo Fisher Scientific, Inc.) and $1 \%$ penicillin/streptomycin. To sort the LSC subpopulation,cells were separated and enriched for $\mathrm{CD} 34^{+} \mathrm{CD} 38^{-}$sub-population using magnetic microbeads (Miltenyi Biotec, Inc.). PE-labelled CD34 (CD34-PE), FITC-labelled CD38 (CD38-FITC) or specific isotype control antibodies were bought from Abcam. In total, eight newly diagnosed and untreated patients with AML (6 males and 2 females) ranging from 3-13 years (median age of 8 years) were involved in the present study and LSC subpopulation was separated from peripheral blood samples. As inclusion criteria, all patients with AML classified as the M1 or M2 French-American-British (FAB) subtypes were included (22), and all other patients were excluded. Written informed consent was obtained from all the parents of the patients in this study. The samples were obtained from the West China Second University Hospital of Sichuan University (Chengdu, China) between September 2019 and October 2019. The study was approved by the Institutional Ethics Committee of the West China Second University Hospital of Sichuan University (Chengdu, China). The same sorting procedure as aforementioned was performed.

For hypoxia exposure, LSCs were cultured in a gas generator system (Model MCO 18 M; Sanyo Biomedical Electrical Co.; https://www.panasonic.com/global/home.html) containing $1 \% \mathrm{O}_{2}, 5 \% \mathrm{CO}_{2}$ and $94 \% \mathrm{~N}_{2}$ for $48 \mathrm{~h}$. As the normoxic control, LSCs were cultured in $20 \% \mathrm{O}_{2}, 5 \% \mathrm{CO}_{2}$ and $75 \% \mathrm{~N}_{2}$ for $48 \mathrm{~h}$. For $\mathrm{CoCl}_{2}$ and echinomycin treatment, LSCs were cultured in original medium supplemented with $125 \mathrm{nM} \mathrm{CoCl}_{2}$ or $200 \mathrm{nM}$ echinomycin in normoxic or hypoxic condition for $0-48 \mathrm{~h}$.

Western blotting. Total protein was extracted using a SoniConvert ${ }^{\circledR}$ sonicator (DocSense; http://www.doc-sense. com/index.html) following the manufacturer's instruction at room temperature for $3 \mathrm{sec}$ and concentration was measured using a BCA assay (Sigma Aldrich; Merck KGaA) and $20 \mu \mathrm{g}$ total protein was loaded per well onto a $6-10 \%$ gradient polyacrylamide gel and resolved using SDS-PAGE. Then the fractionated proteins were transferred to a PVDF membrane (EMD Millipore). Then membrane was blocked with blocking buffer $(0.1 \%$ Tween-20 in $5 \%$ skimmed milk) at room temperature for $0.5 \mathrm{~h}$. The primary antibodies used were are follows: Anti-HIF-1 $\alpha$ (cat. no. ab16066, diluted in 1:1,000), anti-BMI-1 (cat. no. ab38295, diluted in 1:1,000), anti- $\beta$-actin (cat. no. ab8226, diluted in 1:2,000), anti-cleaved caspase-3 (cat. no. ab2302, diluted in 1:1,000), anti-PI3K p85 (cat. no. ab180967, diluted in 1:1,000), anti-PI3K antibody (cat. no. ab32089; 1:1,000), anti-pan-AKT (phosphorylated T308) (cat. no. ab38449; 1:1,000), anti-pan-AKT (cat. no. ab8805; 1:1,000), anti-E cadherin (cat. no. ab1416; 1:1,000) and anti-vimentin (cat. no. ab193555; 1:1,000) were all bought from Abcam. Then, the membrane was washed with $0.1 \%$ Tween-20 Tris-buffered saline and incubated with horseradish peroxidase (HRP)-conjugated secondary antibodies (goat anti-rabbit IgG H\&L; cat. no. ab6721; 1:10,000) for $0.5 \mathrm{~h}$ at room temperature. The bound antibodies were detected using a ECL detection kit (EMD Millipore). $\beta$-actin was used as an internal control. The density of the bands was analyzed using the Quantity One software version 4.3.1 (Bio-Rad Laboratories, Inc.).

Luciferase reporter assay. The dual-luciferase reporter assay kit (Promega Corporation) was employed to evaluate the interaction between hypoxia- or $\mathrm{CoCl}_{2}$-induced HIF-1 $\alpha$ and BMI-1 promoter region. The sequences of the promoter region $(-877$ to +23 bp upstream of transcriptional start point) of BMI-1 was cloned into the luciferase reporter pGL4.11 (Promega, Corporation). In total, $1 \times 10^{5} \mathrm{LSCs}$ sorted from KG-1 $\alpha$ cells were cultured in 12 -well plates, transfected with BMI-1 promoter region (Beyotime Institute of Biotechnology), and then cells were exposed to hypoxia or $\mathrm{CoCl}_{2}$ according to the kit manufacturer's protocol (23). Luciferase activity was measured $48 \mathrm{~h}$ after transfection using the Dual-Luciferase Reporter Assay system (Beyotime Institute of Biotechnology) and firefly luciferase readings were normalized to the activity of Renilla luciferase enzyme.

Cell Counting Kit (CCK)-8 assay. To detect cell viability, $\sim 5.0 \times 10^{3}$ cells were seeded into each well of a 96-well plate. In total, $10 \mu$ l CCK-8 (Sigma-Aldrich; Merck KGaA) was added to each well and incubated for $4 \mathrm{~h}$. Cell viability was evaluated based on absorbance at $490 \mathrm{~nm}$ in a microplate reader (Synergy 2 Multi-Mode Microplate Reader; BioTek Instruments, Inc.). Inhibitory concentration (IC) was calculated according to the following formula: $\%$ inhibition $=[1-($ Absorbance of treatments/absorbance of DMSO) x100]. $\mathrm{IC}_{30}$ and $\mathrm{IC}_{50}$ values of Ara-c, which represent the concentration of Ara-c that is required for 30 or 50 inhibition in vitro, were marked on $\mathrm{X}$ and $\mathrm{Y}$ axis respectively. The combination index of each treatment was calculated according to the classic isobologram equation combination index $=\left[(\mathrm{D})_{1} /(\mathrm{Dx})_{1}\right]+\left[(\mathrm{D})_{2} /(\mathrm{Dx})_{2}\right](24)$. The percent inhibition was calculated according to the following formula: $\%$ Inhibition $=[1-($ absorbance of treatments/absorbance of DMSO) x100]. 
Apoptosis assay. To detect total apoptosis, cells were collected and incubated with $5 \mu \mathrm{l}$ binding reagent and $5 \mu \mathrm{l}$ Annexin V-FITC (BD Biosciences). After 30-min incubation, cells were washed three times with PBS and stained with $5 \mu$ l propidium iodide (PI; Sigma Aldrich; Merck $\mathrm{KGaA}$ ) for $15 \mathrm{~min}$ at room temperature according to the manufacturer's instructions. The lethal dose (LD)50 was defined as the concentration of the drug required to induce cell death by $50 \%$. LD50 was calculated by detecting the apoptosis rate after treatment with different Ara-c concentrations. Briefly, inhibition rate was calculated according to the following formula $=[1$ - $($ death rate of treatments $/$ death rate of DMSO) x100]. LD50 was the concentration that induces 50\% inhibiting rate. The experiments were repeated three times. All data were analysed and calculated using FlowJo software 7.2.4 (Tree Star, Inc.).

Small interfering (si)RNA transfection. To knock down the expression of BMI-1 in LSCs, the BMI-1-specific siRNA was synthesized by Guangzhou RiboBio Co., Ltd. The sequences of the siRNAs used to suppress BMI-1 were as follows: forward, 5'-GCGGUAACCACCAAUCUUCdTdT-3' and reverse, 3'-dTdTCGCCAUUGGUGGUUAGAAG-5', which targeted the sequence of 5'-GCGGTAACCACCAAT CTTC-3'. The scrambled siRNA sequences were employed as negative control as follows: forward, 5'-GACAUGAuC UCCGACCAUCdTdT-3' and reverse, 3'-dTdTCGCGUGAG GUUGAAUCGAU-5'. The siRNA or scrambled siRNA were mixed with Lipofectamine ${ }^{\circledR} 2000$ (Invitrogen; Thermo Fisher Scientific, Inc.) according to the manufacturer's instructions. After transfection for $72 \mathrm{~h}$, total protein and RNAs were prepared from the cells using TRIzol ${ }^{\circledR}$ reagent (Invitrogen; Thermo Fisher Scientific, Inc.) following manufacturer's instructions, and were subjected to western blot analysis and quantitative PCR (qPCR) by using BlazeTaq ${ }^{\mathrm{TM}} \mathrm{SYBR}^{\circledR}$ Green qPCR Mix 2.0 kit (Guangzhou RiboBio Co., Ltd.), respectively. The primers for detecting BMI-1 and $\beta$-actin mRNA were descripted as follows: BMI-1, forward, 5'-CCACCTGAT GTGTGTGCTTG-3' and reverse, 5'-TTCAGTAGTGGTCTG GTCTTGT-3'; $\beta$-actin, forward, 5'-CATGTACGTTGCTAT CCAGGC-3' and reverse, 5'-CTCCTTAATGTCACGCAC GAT-3'. The concentration of purified RNA was determined by the UV spectrophotometer (Invitrogen; Thermo Fisher Scientific, Inc.). CDNA was reversibly transcribed from the extracted total RNA using a Reverse Transcriptase kit (cat. no.: QP006; Guangzhou RiboBio Co., Ltd.) according to the manufacturer's protocol. The PCR reaction was performed on an Applied Biosystems 7500 Real-Time System (ABI 7500HT instrument). Thermocycling conditions were also follows: $5 \mathrm{~min}$ At $50^{\circ} \mathrm{C}, 2 \mathrm{~min}$ at $95^{\circ} \mathrm{C}$ and 40 cycles of $10 \mathrm{sec}$ at $95^{\circ} \mathrm{C}$ and $60 \mathrm{~s}$ at $60^{\circ} \mathrm{C}$. Experiments were performed in triplicate. The qPCR results were analyzed and expressed relative to the threshold cycle $(\mathrm{Cq})$ values, and were then converted to fold-change values; all data was analyzed using $2^{-\Delta \Delta \mathrm{Cq}}$ method (25). A 2.0-fold change was considered to be significant (25). In total, three repeats were conducted for each sample.

Colony formation in soft agar. For the soft agar assay, $1 \times 10^{3}$ LSCs were mixed with $0.3 \%$ low melting agar in
RPMI-1640 medium supplemented with 10\% FBS and plated on a $0.6 \%$ low melting agar-coated 6 -well plate. The plates were incubated at $37^{\circ} \mathrm{C}$ in a humidified incubator containing $5 \% \mathrm{CO}_{2}$ for 2 weeks. Every well was stained with $0.2 \mathrm{ml}$ $0.05 \%$ crystal violet for $0.5 \mathrm{~h}$ at $37^{\circ} \mathrm{C}$. The numbers of positive colonies ( $>8$ cells/colony) were counted. The experiments were performed at least three times.

Statistical analysis. Statistical analysis was performed using SPSS version 19.0 (IBM Corp.). The data are presented as the mean $\pm \mathrm{SD}$, unless otherwise shown. A two-tailed unpaired Student's t-test was used for statistical analysis. ANOVA was performed to compare multiple groups with one variable followed by Tukey's post hoc test. $\mathrm{P}<0.05$ was considered to indicate a statistically significant difference. The experiments were repeated at least three times.

\section{Results}

Hypoxia exposure induces BMI-1 expression in LSCs. To obtain LSCs from KG-1 $\alpha$ and primary AML cells, cells were stained with anti-CD38 PE-conjugated antibody and anti-CD34 FITC-conjugated antibody and sorted using flow cytometry, then the proportion of CD34+CD38- was measured. As shown in Fig. 1A, in post-sorted cells of KG-1 $\alpha$, the proportion of CD34+CD38- (33.1 $\pm 0.9 \%$, in quadrant 3$)$ was significantly higher compared with that in pre-sorted cells $(1.3 \pm 0.3 \%$, in quadrant 3 ). In post-sorted cells of primary AML, the proportion of CD34+CD38- $(54.3 \pm 0.7 \%$, in quadrant 3$)$ was also higher compared with that in pre-sorted cells $(0.6 \pm 0.3 \%$, in quadrant 3). Cell cycle analysis then demonstrated that there was no significant difference in proliferation between pre-sorted and post-sorted cells (Fig. 1B).

Enriched LSCs were exposed to hypoxia from $0-48 \mathrm{~h}$ and then analysed using western blotting. The results demonstrated that hypoxia significantly induced HIF-1 $\alpha$ and BMI-1 protein levels in LSCs after 18-h exposure to hypoxia (Fig. 2A). As shown in Fig. 2A, the steady-state level of BMI-1 increased at $6 \mathrm{~h}$, reached a peak at $18 \mathrm{~h}$, and was sustained at least to $48 \mathrm{~h}$ after hypoxia exposure. Expectedly, upregulation of HIF-1 $\alpha$ was also observed after hypoxia exposure. To confirm whether hypoxia upregulates HIF-1 $\alpha$ and BMI-1 in primary AML LSCs, eight LSC clones were exposed to hypoxia for $24 \mathrm{~h}$. Consistently, both HIF-1 $\alpha$ and BMI-1 were notably upregulated (Fig. 2B). This indicated that the effect of hypoxia on HIF-1 $\alpha$ and BMI-1 were long-lasting, and so further analyses using LSCs sorted from KG-1 $\alpha$ cells were performed.

BMI-1 is transcriptionally activated by HIF-1 $\alpha$ induction after hypoxia exposure (22). To confirm whether BMI-1 is transcriptionally regulated by $\mathrm{HIF}-1 \alpha, 125 \mathrm{nM} \mathrm{CoCl}_{2}$ was added to induce HIF-1 $\alpha$ under normoxic conditions and $200 \mathrm{nM}$ echinomycin was employed to abolish HIF-1 $\alpha$ transcriptional activity. As it is shown in Fig. 2C, both hypoxia and $\mathrm{CoCl}_{2}$ exposure upregulated HIF-1 $\alpha$ and BMI-1, and addition of $200 \mathrm{nM}$ echinomycin disturbed the upregulation of BMI-1 without affecting HIF-1 $\alpha$, indicating that transcriptional activity of HIF1- $\alpha$ is responsible for the regulation of BMI-1 after hypoxia and $\mathrm{CoCl}_{2}$ exposure. To further confirm whether hypoxia-induced BMI-1 is mediated by HIF-1 $\alpha$, luciferase 
A
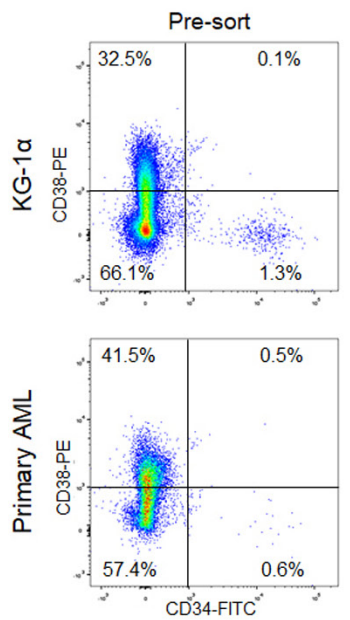
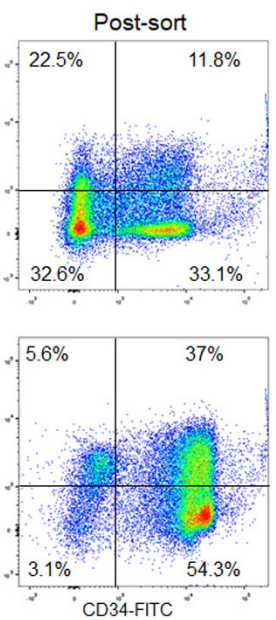

- $\mathrm{CD} 34+\mathrm{CD} 38-$

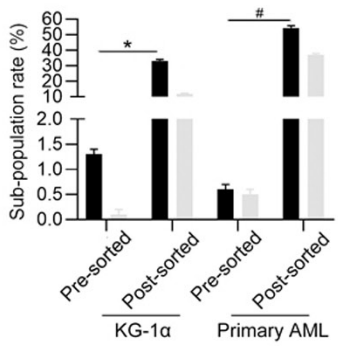

B

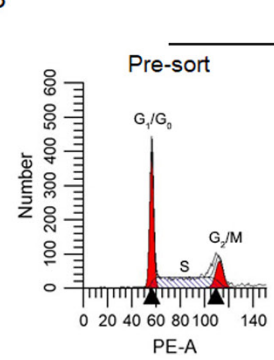

KG-1 $\alpha$

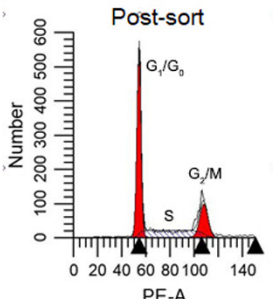

PE-A

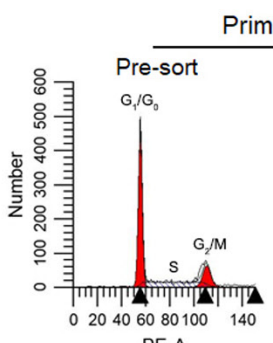

PE-A

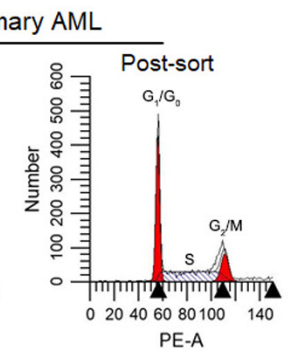

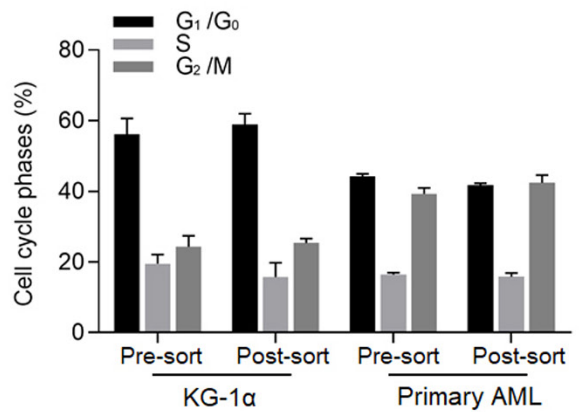

Figure 1. Enrichment of CD34+CD38- cells from KG-1 $\alpha$ cells and primary AML (A) Sorting of CD34+/CD38- cells from KG-1 $\alpha$ and primary AML. (B) Cell cycle analysis of pre-sorted and post-sorted cells. ${ }^{*} \mathrm{P}<0.05$, vs. pre-sorted KG-1 $\alpha$ cell group; ${ }^{*} \mathrm{P}<0.05$ vs. post-sorted cell group. AML, acute myeloid leukaemia.

reporter plasmids were constructed containing the BMI-1 promoter region ( -877 to $+23 \mathrm{bp}$; Fig. 2D) and tested the effects of HIF-1 $\alpha$ on their activity. Both hypoxia and $\mathrm{CoCl}_{2}$ exposure increased luciferase activity compared with the normoxia group, and are reversed by co-culturing with $200 \mathrm{nM}$ echinomycin. These findings suggested that BMI-1 upregulated by hypoxia was transcriptionally dependent on HIF-1 $\alpha$. All these data indicated that hypoxia exposure transcriptionally upregulated BMI-1 via HIF-1 $\alpha$ in LSCs.

Hypoxia induces chemoresistance in LSCs. To determine the effect of hypoxia exposure on chemosensitivity of LSCs, 0-4.0 $\mu \mathrm{M}$ Ara-C was supplemented in culture medium and cell viability was measured $24 \mathrm{~h}$ later. As it is shown in Fig. 3A (left panel), hypoxia exposure significantly inhibited the decrease of cell viability compared with the normoxia group, which was reversed by $200 \mathrm{nM}$ echinomycin co-treatment. It is also observed that $\mathrm{CoCl}_{2}$-exposed LSCs presented the same tendency (Fig. 3A, right panel), indicating the potential involvement of HIF-1 $\alpha$. Then, the apoptosis induced by $1 \mu \mathrm{M}$ Ara-C after $24 \mathrm{~h}$ was analysed using Annexin V-FITC/PI double staining and western blotting for cleaved caspase-3. In Fig. $3 \mathrm{~B}$, it is observed that $1 \mu \mathrm{M}$ of Ara-C treatment induced $12.54 \pm 0.85 \%$ total apoptosis in normoxia group and $13.79 \pm 1.22 \%$ total apoptosis in Mock group, and hypoxia $(7.92 \pm 0.47 \%)$ and $\mathrm{CoCl}_{2}$ exposure $(4.86 \pm 0.52 \%)$ decreased total apoptosis induced by $1 \mu \mathrm{M}$ of Ara-C treatment in LSCs, which was reversed by echinomycin co-treatment (Fig. 3B, left panel). Western blotting also confirmed that cleaved caspase-3 was decreased by hypoxia and $\mathrm{CoCl}_{2}$ exposure (Fig. 3B, right panel).

Hypoxia-induced BMI-1 activates the PI3K/Akt pathway and is partially responsible for chemoresistance to Ara-C in LSCs. BMI-1 is reported to activate the PI3K/Akt pathway and thus causes chemoresistance $(26,27)$. Therefore, the present study investigated the role of hypoxia-induced BMI-1 in regulating the PI3K/Akt pathway and chemoresistance. Firstly, the high efficacy of siBMI-1-mediated knockdown of BMI-1 protein level was confirmed (Fig. 4A). Expectedly, addition of echinomycin failed to affect HIF-1 $\alpha$ protein level. By performing western blotting, it was demonstrated that phosphorylated PI3K and Akt were increased after hypoxia exposure and decreased by BMI-1-knockdown and inhibition of HIF-1 $\alpha$ transcriptional activity (Fig. 4B). Notably, siBMI1 significantly decreased the levels of phosphorylated Akt, via decreasing total Akt protein level at least partially. As expected, hypoxia-induced chemoresistance and total apoptosis (10.83 $\pm 0.45 \%$ in Hypoxia+siScrambled group) are notably reversed by BMI-1-knockdown $(15.40 \pm 1.33 \%)$ and

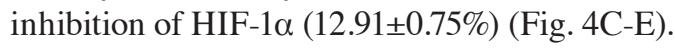

Hypoxia-induced BMI-1 promotes EMT and inhibits self-renewal of LSCs under hypoxic conditions. BMI-1 is reported to regulate EMT in several other kinds of cancer (28-30), therefore, the present study analysed the effect of hypoxia-induced BMI-1 on EMT. Expectedly, hypoxia exposure downregulated E-cadherin and upregulated vimentin 
A
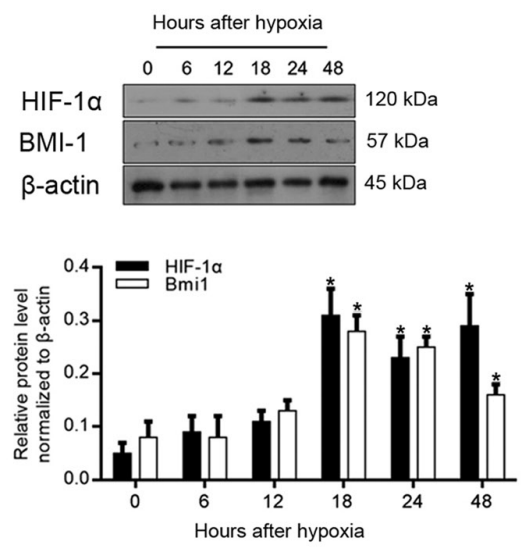

C
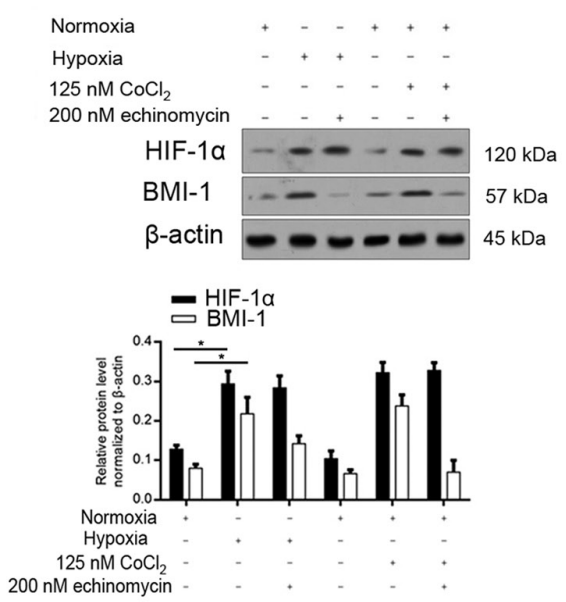

B

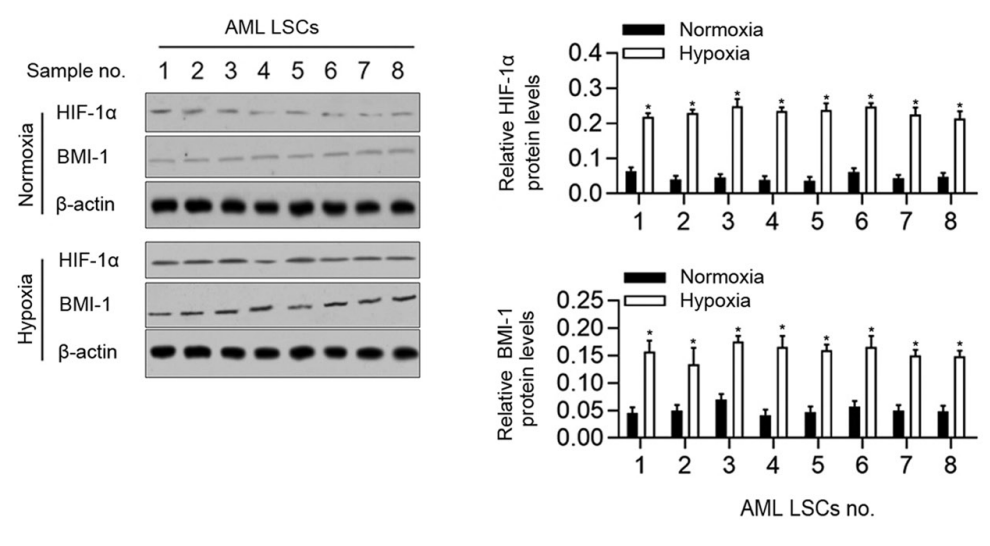

D

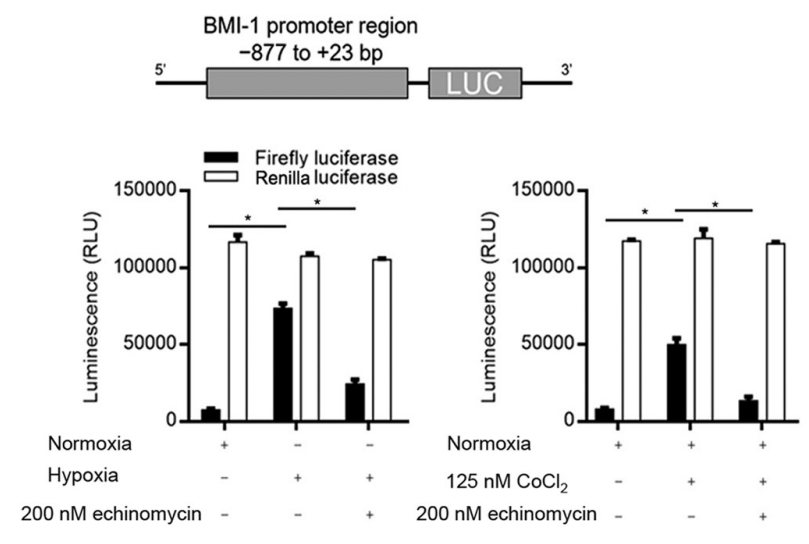

Figure 2. Hypoxia exposure induces BMI-1 expression in LSCs sorted from KG-1 $\alpha$ cells. (A) After hypoxia exposure for 0-48 h, the protein levels of HIF-1 $\alpha$ and BMI-1 were measured by semi-quantitative western blotting in LSCs sorted from KG-1 $\alpha$. (B) After hypoxia exposure, eight LSCs sorted from primary AML samples were detected for HIF-1 $\alpha$ and BMI-1 protein levels. (C) HIF-1 $\alpha$ and BMI-1 were measured after hypoxia and $\mathrm{CoCl}_{2}$ exposure with or without $200 \mathrm{nM}$ echinomycin for $48 \mathrm{~h}$ in LSCs sorted from KG-1 $\alpha$. (D) Relative luciferase activity of the BMI-1 promoter reporter genes was measured in LSCs sorted from KG-1 $\alpha$. "P $<0.05$ vs. $0 \mathrm{~h}$ or normoxia group, ${ }^{, \mathrm{P}}<0.05$, vs. $0 \mathrm{~h}$ or hypoxia group, ${ }^{*} \mathrm{P}<0.05$, vs. $\mathrm{CoCl}_{2}$ treated group. LSCs, leukaemia stem cells; HIF-1 $\alpha$, hypoxia-inducible factor-1 $\alpha$; BMI-1, B cell-specific Moloney murine leukaemia virus integration site 1; AML, acute myeloid leukaemia; LUC.

compared with the normoxia group, which were notably reversed by BMI-1-knockdown and HIF-1 $\alpha$ inhibition (Fig. 5A). Accordingly, BMI-1 increased the self-renewal capacity of LSCs and promoted EMT under normoxic conditions (31), then BMI-1 was knocked down or HIF-1 $\alpha$ transcriptional activity was inhibited, and colony formation in soft agar was analysed. As expected, hypoxia-induced inhibition in colony formation was significantly increased by BMI-1-knockdown and abolished HIF-1 $\alpha$ transcriptional activity compared with the scrambled control, demonstrating that hypoxia-induced BMI-1 is critical in inhibiting malignance of LSCs (Fig. 5B). Taken together, all these results indicated that hypoxia exposure-induced HIF-1 $\alpha$ tightly regulates EMT and stemness in LSCs via regulating BMI-1.

\section{Discussion}

The present study demonstrated that overexpression of HIF-1 $\alpha$ transcriptionally activates BMI-1, a member of the Polycomb group family of transcription repressors (32), and thus activates the PI3K/Akt pathway and promotes the EMT.
Consequently, hypoxia exposure stimulated HIF-1 $\alpha$ and affected malignant behaviours in LSCs in this manner. It was reported that both hypoxia and $\mathrm{CoCl}_{2}$ exposure-induced HIF-1 $\alpha$ successfully upregulated BMI-1 in LSCs and that such induction is dependent on the transcriptional activity of HIF-1 $\alpha$. Hypoxia notably activated the PI3K/Akt pathway by promoting the phosphorylation of PI3K and Akt, and blockage of HIF-1 $\alpha$ transcriptional activity and knockdown of BMI-1 resulted in blockade of PI3K/Akt, at least in part. It is also observed that hypoxia exposure promoted EMT dependent on the induction of HIF-1 $\alpha$. These observations demonstrated that HIF-1 $\alpha /$ BMI-1 may act as a functional element that serves an important role in regulating the malignant behaviours and self-renewal capacity of LSCs in an hypoxia-modified microenvironment.

BMI-1 is widely overexpressed in several types of cancer, including leukaemia, gastric and lung cancer, and is responsible for promoting malignant behaviours, including transformation, proliferation, invasion, distant metastasis and poor survival (1-4). Moreover, BMI-1 maintains the self-renewal of cancer stem-like cells (CSCs) under 
A
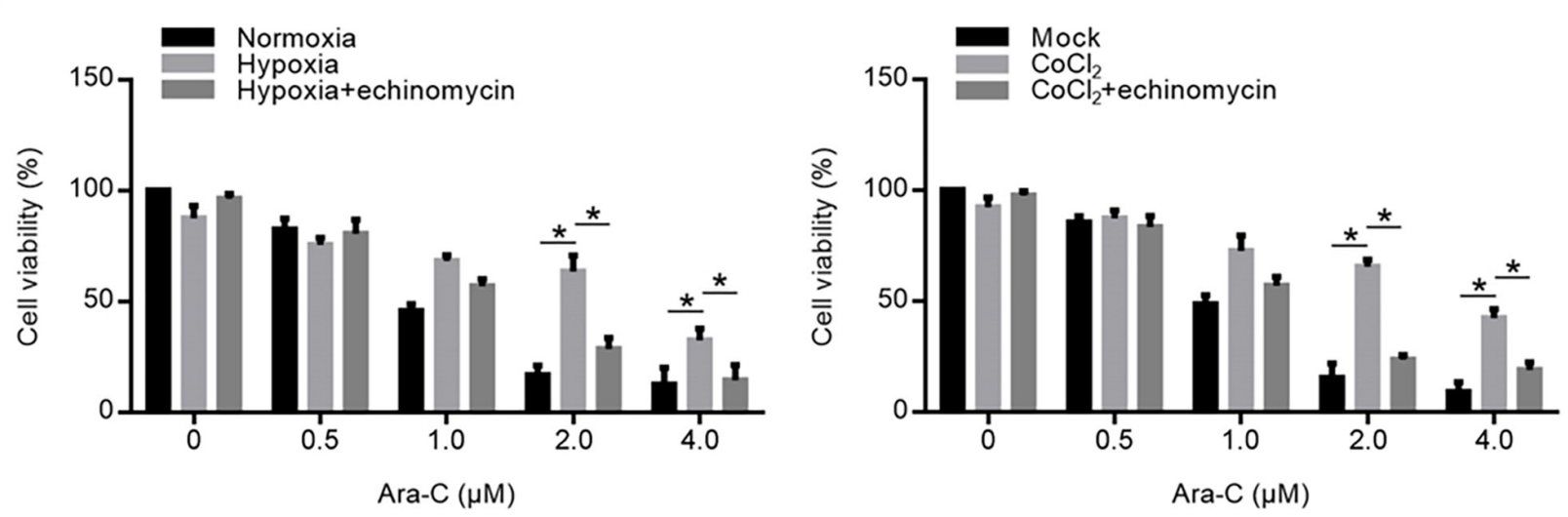

B

$1 \mu \mathrm{M}$ of Ara-C
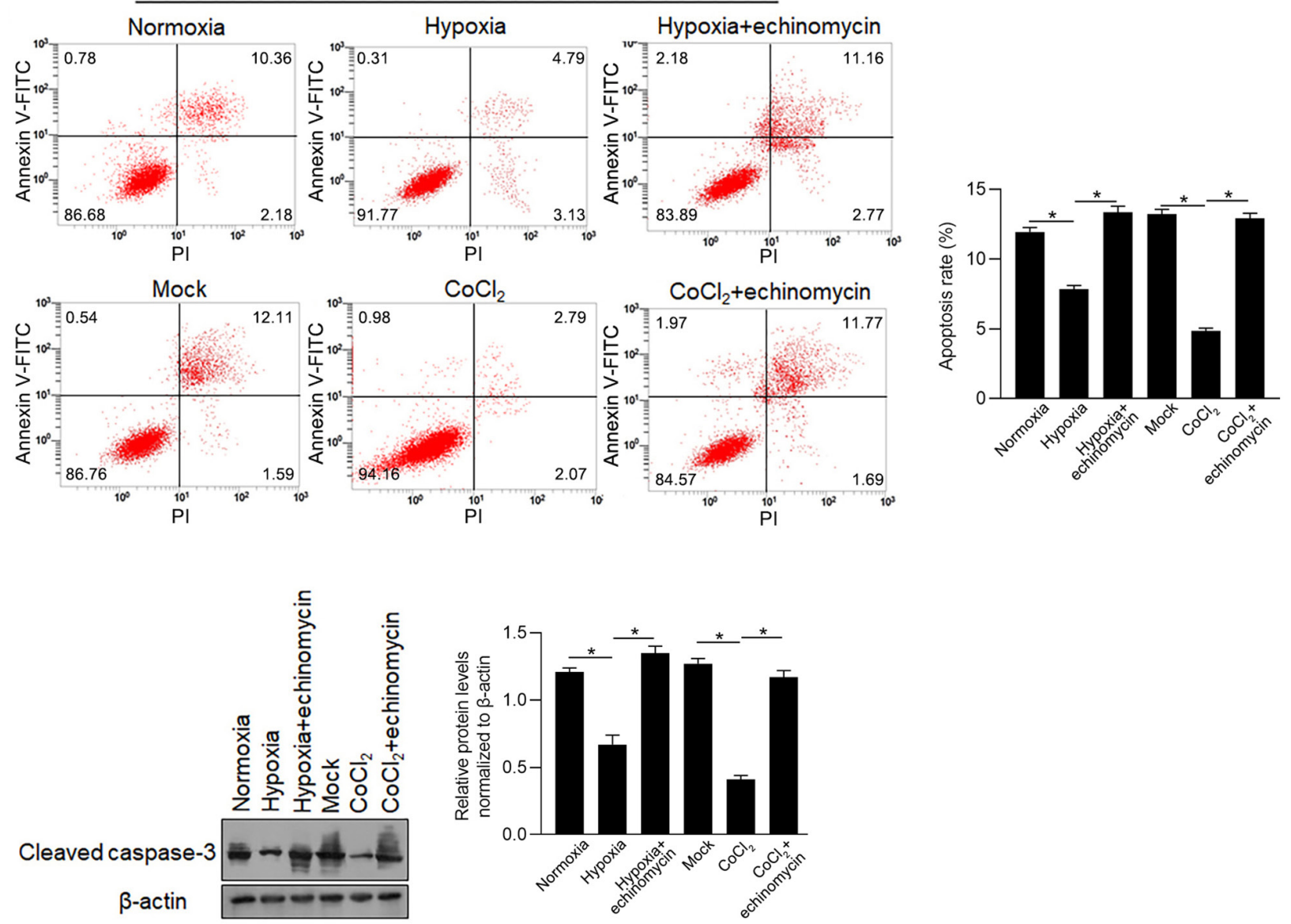

Figure 3. Upregulated HIF-1 $\alpha$ promotes chemoresistance via its transcriptional activity in leukaemia stem cells sorted from KG-1 $\alpha$ cells. (A) After hypoxia or $\mathrm{CoCl}_{2}$ exposure, chemosensitivity to Ara-C was measured after $24 \mathrm{~h}$. (B) After treatment with $1 \mu \mathrm{M}$ Ara-C, Annexin V-FITC and PI double staining was performed followed and apoptosis was analysed using flow cytometry (left panel). Then, cleaved capase-3 was measured using western blotting (right panel). ${ }^{*} \mathrm{P}<0.05$ vs. hypoxia group or $\mathrm{CoCl}_{2}$ group. HIF-1 $\alpha$, hypoxia-inducible factor- $1 \alpha$; Ara-C, cytarabine arabinoside.

normoxic conditions in some tumours, including breast cancer, glioma and leukaemia $(5,6)$. Chiba et al (33) reported that BMI-1 promotes the maintenance of the self-renewal capacity of CSCs enriched from hepatocellular carcinoma. Jin et al (34) reported that, in prostate cancer cells, knockdown of BMI-1 blocked tumorigenic potential and inhibited the cells' self-renewal capacity. According to these studies, BMI-1 acts as a promoter of self-renewal capacity of CSCs under normoxia conditions. The present study aimed to determine the potential effects of BMI-1 under hypoxic conditions, instead of normoxic conditions. Notably, after hypoxia-exposure, it was observed that the stemness of LSCs decreased, which could be reversed by BMI-1-knockdown or inhibition of HIF-1 $\alpha$ transcriptional activity by addition of echinomycin. All these results indicated that BMI-1 may exert different roles under hypoxic compared with normoxic conditions. However, the results only detected the subpopulations of CD34 and CD38, instead of detecting stem cell markers, including NANOG, OCT4 and SOX2 (31), which is a limitation of the present study. 
A

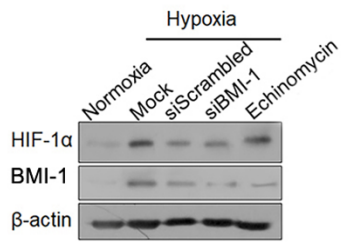

B
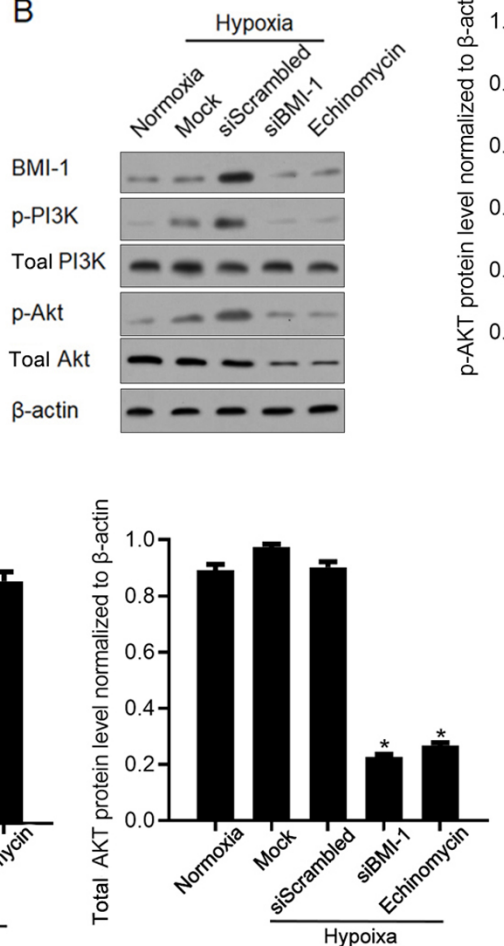
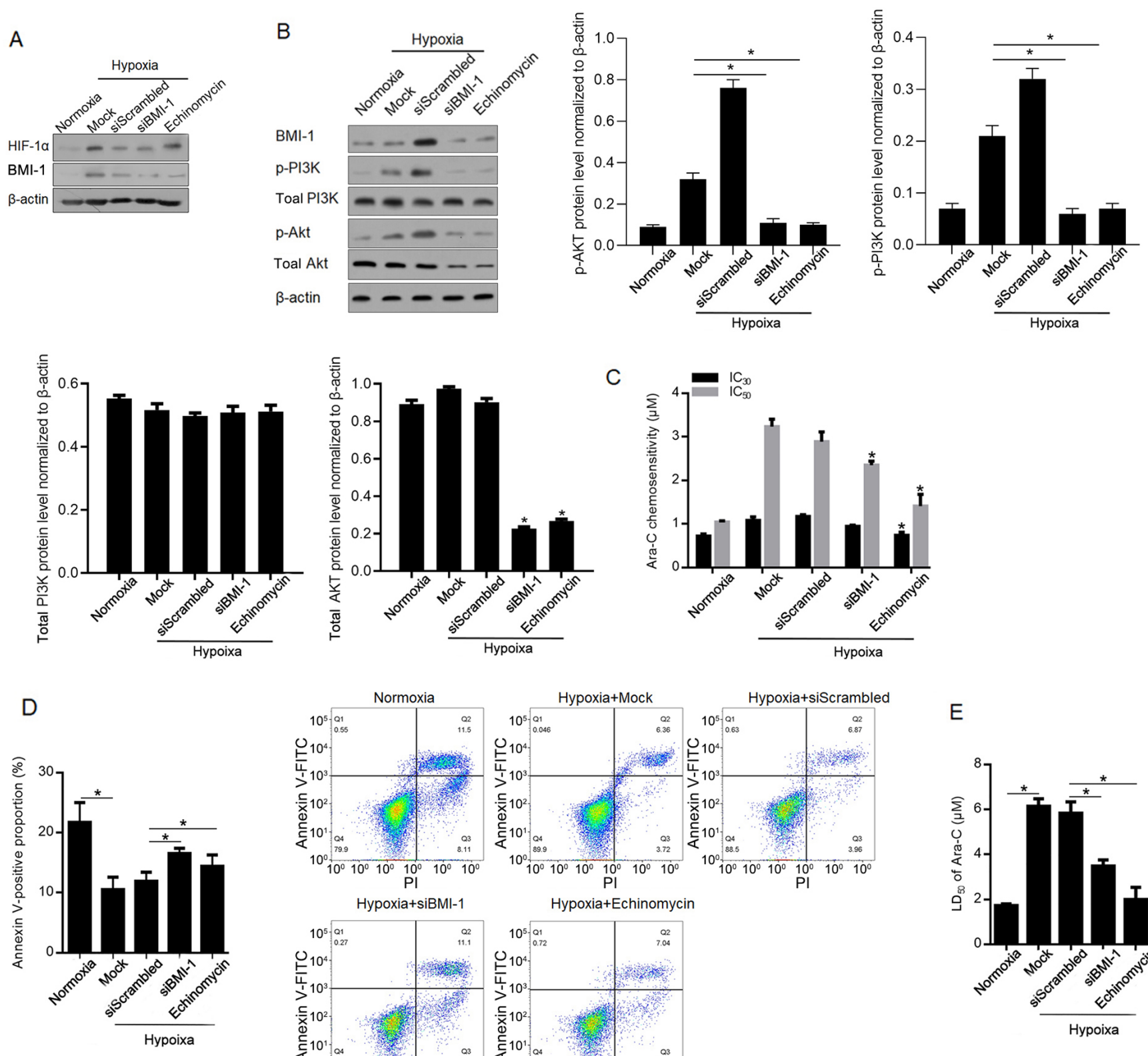
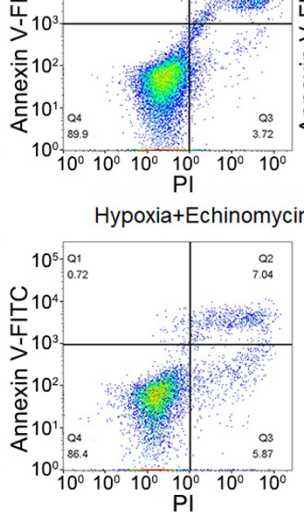

E

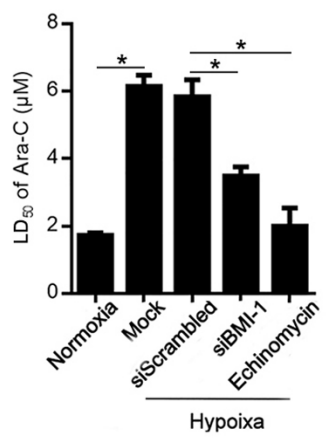

Figure 4. BMI-1 activates the PI3K/Akt pathway, inducing chemoresistance to Ara-C in leukaemia stem cells sorted from KG-1 $\alpha$ cells. (A) After siRNA target to BMI-1 mRNA transfection, the knockdown efficacy of BMI-1 was confirmed using western blotting. (B) Levels of p-PI3K, PI3K, p-Akt and Akt were measured after BMI-1-knockdown. $(\mathrm{C}) \mathrm{IC}_{30}$ and $\mathrm{IC}_{50}$ of Ara-C were measured. (D) Apoptotic rate was measured after $1 \mu \mathrm{M}$ Ara-C treatment. (E) $\mathrm{LD}_{50}$ was measured after Ara-C treatment. "P<0.05, vs. siScrambled group or normoxia group. si, small interfering; BMI-1, B cell-specific Moloney murine leukaemia virus integration site 1; p-, phosphorylated; Ara-C, cytarabine arabinoside; LD, lethal dose; IC, inhibitory concentration.

A

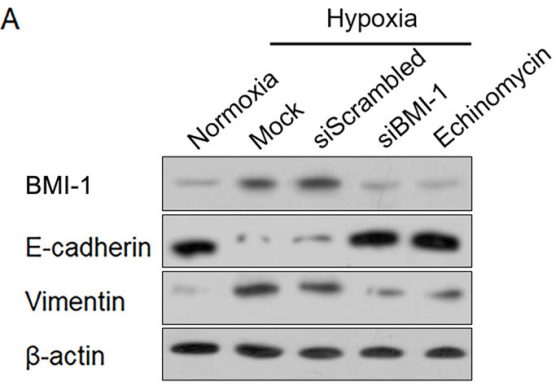

B

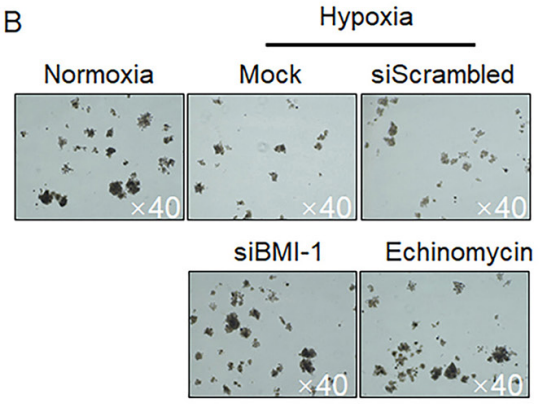

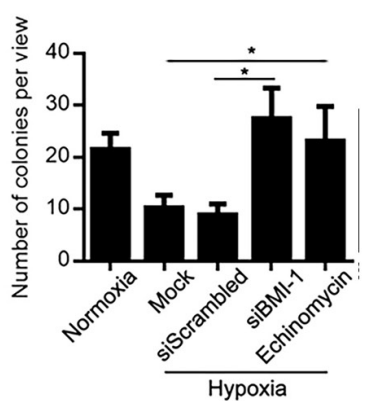

Figure 5. BMI-1 activates the epithelial mesenchymal transition and inhibits the self-renewal capacity of leukaemia stem cells sorted from KG-1 $\alpha$ cells. (A) E-cadherin and vimentin levels were measured using western blotting. (B) Colony formation in soft agar was measured after BMI-1 knockdown. "P<0.05 vs. siScrambled group, ${ }^{*} \mathrm{P}<0.05$ vs. mock group. BMI-1, B cell-specific Moloney murine leukaemia virus integration site 1; si, small interfering. 
Upregulated BMI-1 also induces the EMT, activates the PI3K/Akt pathway and subsequently promotes the aggressiveness of human carcinoma cells, including nasopharyngeal carcinoma (9) and colon cancer (10). The present results demonstrated that transcriptionally upregulated BMI-1 by hypoxia-induced HIF-1 $\alpha$ markedly activated the PI3K/Akt pathway and promoted EMT. HIF-1 $\alpha$, as the most important oxygen-sensitive sub-unit of HIF-1, activates the transcription of numerous genes that regulate several physiological processes, including angiogenesis, cell proliferation and survival, glucose metabolism, $\mathrm{pH}$ regulation and migration in cancer (11). The present study reported that following knockdown of BMI-1 after hypoxia exposure, the malignant behaviours are inhibited, similar to the effect of inhibiting HIF-1 $\alpha$ transcriptional activity. However, it is still unknown whether HIF-1 $\alpha$-induced BMI-1 is necessary for regulating malignant behaviours in LSCs. Another limitation of the present study was that it was not confirmed whether BMI-1 regulates EMT processes under normoxia. It would be valuable to investigate the regulatory role of BMI-1 on EMT processes and stemness maintenance in LSCs, which could provide further information regarding the function of BMI-1 under normoxia.

In summary, the present data demonstrated that hypoxia-induced HIF-1 $\alpha$ transcriptionally upregulated BMI-1 in LSCs. Upregulated BMI-1 regulated malignant behaviours and chemoresistance and inhibited the self-renewal capacity potentially by activating the PI3K/Akt pathway and promoting EMT in LSCs. These findings emphasized that, in a hypoxic microenvironment supporting LSC survival, the hypoxia-activated HIF-1 $\alpha /$ BMI-1 pathway may provide potential opportunities to improve the therapeutic targeting of LSCs.

\section{Acknowledgements}

The authors would like to thank Dr Daiwen Zhao (Chongqing University) for language editing.

\section{Funding}

The present study was funded by The National Science Foundation for Young Scientists of China (grant no. 81600122), The Health and Family Planning Commission of Sichuan Province (grant no. 150106) and The Application Foundation Program of Science and Technology Department of Sichuan Province (grant nos. 19ZDYF1202 and 2020YFS0253).

\section{Availability of data and materials}

The datasets used and/or analysed during the current study are available from the corresponding author on reasonable request.

\section{Authors' contributions}

MYJ, GQH and JG designed detailed protocols and strategies and performed experiments. XG, JHL and JRL were responsible for the data collection and analysis. JHL and JRL interpretated the data. JG supervised the experiments, and was involved in writing and revision of the manuscript. All authors read and approved the final manuscript.

\section{Ethics approval and consent to participate}

Not applicable.

\section{Patient consent for publication}

Not applicable.

\section{Competing interests}

The authors declare that they have no competing interests.

\section{References}

1. Hu Y and Li S: Survival regulation of leukemia stem cells. Cell Mol Life Sci 73: 1039-1050, 2016.

2. Dean M, Fojo T and Bates S: Tumour stem cells and drug resistance. Nat Rev Cancer 5: 275-284, 2005.

3. Linenberger ML, Hong T, Flowers D, Sievers EL, Gooley TA, Bennett JM, Berger MS, Leopold LH, Appelbaum FR and Bernstein ID: Multidrug-resistance phenotype and clinical responses to gemtuzumab ozogamicin. Blood 98: 988-994, 2001.

4. Pei S and Jordan CT: How close are we to targeting the leukemia stem cell? Best Pract Res Clin Haematol 25: 415-418, 2012.

5. She M, Niu X, Chen X, Li J, Zhou M, He Y, Le Y and Guo K: Resistance of leukemic stem-like cells in AML cell line KG1a to natural killer cell-mediated cytotoxicity. Cancer Lett 318: 173-179, 2012.

6. Fuchs D, Daniel V, Sadeghi M, Opelz G and Naujokat C: Salinomycin overcomes ABC transporter-mediated multidrug and apoptosis resistance in human leukemia stem cell-like KG-1a cells. Biochem Biophys Res Commun 394: 1098-1104, 2010.

7. Chen EY, Mazure NM, Cooper JA and Giaccia AJ: Hypoxia activates a platelet-derived growth factor receptor/phosphatidylinositol 3-kinase/Akt pathway that results in glycogen synthase kinase-3 inactivation. Cancer Res 61: 2429-2433, 2001.

8. Zhang H, Li H, Xi HS and Li S: HIF1 $\alpha$ is required for survival maintenance of chronic myeloid leukemia stem cells. Blood 119: 2595-2607, 2012.

9. Majmundar AJ, Wong WJ and Simon MC: Hypoxia-inducible factors and the response to hypoxic stress. Mol Cell 40: 294-309, 2010.

10. Hermitte F, Brunet de la Grange P, Belloc F, Praloran V and Ivanovic Z: Very low $\mathrm{O} 2$ concentration $(0.1 \%)$ favors $\mathrm{G} 0$ return of dividing CD34 ${ }^{+}$cells. Stem Cells 24: 65-73, 2006.

11. Rizo A, Dontje B, Vellenga E, de Haan G and Schuringa JJ: Long-term maintenance of human hematopoietic stem/progenitor cells by expression of BMI1. Blood 111: 2621-2630, 2008.

12. Iwama A, Oguro H and Negishi M, Kato Y, Morita Y, Tsukui H, Ema H, Kamijo T, Katoh-Fukui Y, Koseki H, et al: Enhanced self-renewal of hematopoietic stem cells mediated by the polycomb gene product Bmi-1. Immunity 21: 843-851, 2004.

13. Lessard J and Sauvageau G: Bmi-1 determines the proliferative capacity of normal and leukaemic stem cells. Nature 423: 255-260, 2003.

14. Park IK, Qian D, Kiel M, Becker MW, Pihalja M, Weissman IL, Morrison SJ and Clarke MF: Bmi-1 is required for maintenance of adult self-renewing haematopoietic stem cells. Nature 423: 302-305, 2003.

15. Siddique HR and Saleem M: Role of BMI1, a stem cell factor, in cancer recurrence and chemoresistance: Preclinical and clinical evidences. Stem Cells 30: 372-378, 2012.

16. Meng S, Luo M, Sun H, Yu X, Shen M, Zhang Q, Zhou R, Ju X, Tao W, Liu D, et al: Identification and characterization of Bmi-1-responding element within the human p16 promoter. J Biol Chem 285: 33219-33229, 2010.

17. Saudy NS, Fawzy IM, Azmy E, Goda EF, Eneen A and Abdul SE: BMI1 gene expression in myeloid leukemias and its impact on prognosis. Blood Cells Mol Dis 53: 194-198, 2014.

18. Cerami E, Gao J, Dogrusoz U, Gross BE, Sumer SO, Aksoy BA, Jacobsen A, Byrne CJ, Heuer ML, Larsson E, et al: The cBio cancer genomics portal: An open platform for exploring multidimensional cancer genomics data. Cancer Discov 2: 401-404, 2012. 
19. Ye H, Adane B, Khan N, Sullivan T, Minhajuddin M, Gasparetto M, Stevens B, Pei S, Balys M, Ashton JM, et al: Leukemic stem cells evade chemotherapy by metabolic adaptation to an adipose tissue niche. Cell Stem Cell 19: 23-37, 2016.

20. Kreso A, van Galen P, Pedley NM, Lima-Fernandes E, Frelin C, Davis T, Cao L, Baiazitov R, Du W, Sydorenko N, et al: Self-renewal as a therapeutic target in human colorectal cancer. Nat Med 20: 29-36, 2014.

21. Nishida Y, Maeda A, Kim MJ, Cao L, Kubota Y, Ishizawa J, AlRawi A, Kato Y, Iwama A, Fujisawa M, et al: The novel BMI-1 inhibitor PTC596 downregulates MCL-1 and induces p53-independent mitochondrial apoptosis in acute myeloid leukemia progenitor cells. Blood Cancer J 7: e527, 2017.

22. Bennet JM, Catovsky D, Daniel MT, Flandrin G, Galton DA, Gralnick HR and Sultan C: Proposal for the classification of acute leukaemias. French-American-British (FAB) Co-Operative Group. Br J Haematol 33: 451-458, 1976.

23. Du R, Xia L, Ning X, Liu L, Sun W, Huang C, Wang H and Sun S: Hypoxia-induced Bmil promotes renal tubular epithelial cell-mesenchymal transition and renal fibrosis via PI3K/Akt signal. Mol Biol Cell 25: 2650-2659, 2014.

24. Chou TC and Talalay P: Analysis of combined drug effects: A new look at a very old problem. Trends Pharmacol Sci 4 450-454, 1983

25. Livak KJ and Schmittgen TD: Analysis of relative gene expression data using real-time quantitative PCR and the 2(-Delta Delta $\mathrm{C}(\mathrm{T})$ ) method. Methods 25: 402-408, 2001.

26. Wu Z, Min L, Chen D, Hao D, Duan Y, Qiu G and Wang Y: Overexpression of BMI-1 promotes cell growth and resistance to cisplatin treatment in osteosarcoma. PLoS One 6: e14648, 2011.

27. Zhang Z, Bu X, Chen H, Wang Q and Sha W: Bmi-1 promotes the invasion and migration of colon cancer stem cells through the downregulation of E-cadherin. Int J Mol Med 38: 1199-1207, 2016.

28. Li H, Song F, Chen X, Li Y, Fan J and Wu X: Bmi-1 regulates epithelial-to-mesenchymal transition to promote migration and invasion of breast cancer cells. Int J Clin Exp Pathol 7: 3057-3064, 2014.
29. Yuan W, Yuan Y,Zhang T and Wu S: Role of Bmi-1 in regulation of ionizing irradiation-induced epithelial-mesenchymal transition and migration of breast cancer cells. PLoS One 10: e118799, 2015.

30. Jiang L, Wu J, Yang Y, Liu L, Song L, Li J and Li M: Bmi-1 promotes the aggressiveness of glioma via activating the NF-kappaB/MMP-9 signaling pathway. BMC Cancer 12: 406, 2012.

31. Paranjape AN, Balaji SA, Mandal T, Krushik EV, Nagaraj P, Mukherjee G and Rangarajan A: Bmil regulates self-renewal and epithelial to mesenchymal transition in breast cancer cells through Nanog. BMC Cancer 14: 785, 2014.

32. Alkema MJ, Wiegant J, Raap AK, Berns A and van Lohuizen M: Characterization and chromosomal localization of the human proto-oncogene BMI-1. Hum Mol Genet 2: 1597-1603, 1993.

33. Chiba T, Miyagi S, Saraya A, Aoki R, Seki A, Morita Y, Yonemitsu Y, Yokosuka O, Taniguchi H, Nakauchi $\mathrm{H}$ and Iwama A: The polycomb gene product BMI1 contributes to the maintenance of tumor-initiating side population cells in hepatocellular carcinoma. Cancer Res 68: 7742-7749, 2008.

34. Jin M, Zhang T, Liu C, Badeaux MA, Liu B, Liu R, Jeter C, Chen X, Vlassov AV and Tang DG: miRNA-128 suppresses prostate cancer by inhibiting BMI-1 to inhibit tumor-initiating cells. Cancer Res 74: 4183-4195, 2014.

c) (7) $\odot$ This work is licensed under a Creative Commons cC Atribution-NonCommercial-NoDerivatives 4.0 International (CC BY-NC-ND 4.0) License. 